\title{
FLUX TUBE SHREDDING AND ITS INFRARED SIGNATURE
}

\author{
M. BÜNTE
}

Institute of Astronomy, ETH Zentrum, CH-8092 Zürich, Switzerland

O. STEINER

Kiepenheuer-Institut für Sonnenphysik, Schöneckstrasse 6, D-7800 Freiburg, FRG

S.K. SOLANKI

Institute of Astronomy, ETH Zentrum, CH-8092 Zürich, Switzerland

and

V.J. PIZZO

San Juan Capistrano Research Institute, San Juan Capistrano, CA, U.S.A.

\begin{abstract}
The interchange instability of solar magnetic flux tubes and possible stabilization mechanisms are reviewed. Special attention is paid to the influence of magnetic tension forces and the internal atmosphere, both of which were neglected in earlier studies of this instability. It is found that whirl flows with velocities of only $2.2 \mathrm{~km} \mathrm{~s}^{-1}$ are strong enough to stabilize the flux tubes. However, their absence or the excitation of other instabilities might lead to a shredding of the tubes. The observability of such a scenario in the infrared is briefly discussed.
\end{abstract}

Key words: infrared: stars - MHD - Sun: magnetic fields

\section{Introduction}

Vertical magnetic flux tubes fan out with height due to the decreasing gas pressure of the stratified solar atmosphere. Concave magnetic configurations, however, are generally unstable to the interchange instability and should therefore easily break apart into smaller flux tubes, i.e., become shredded. In contrast, the observed lifetimes of sunspots (weeks to months) and small magnetic elements (18 minutes or more) exceed the growth time of the instability by far $(\sim 1 \mathrm{~h}$ for sunspots, $\sim 20 \mathrm{~s}$ for small tubes), indicating that there exists a quasi-static phase in the life of magnetic flux tubes on the Sun. Meyer et al. (1977) found that tubes are stabilized by buoyancy forces if their magnetic fluxes exceed the critical limit of $10^{19}-10^{20} \mathrm{Mx}$. Schüssler (1984) suggested that smaller tubes could be stabilized by whirl flows, which are expected to arise from the "bathtub effect" in granular downdrafts. However, for a certain range of fluxes $\left(5 \times 10^{17}-10^{19} \mathrm{Mx}\right)$ the whirl velocities found in Schüssler's analysis are unrealistically high (i.e., well above $2 \mathrm{~km} \mathrm{~s}^{-1}$ ) which led him to suggest that tubes with radii of $250-1,100 \mathrm{~km}\left(\right.$ at $\left.\tau_{5000}=1, B \approx 1,600 \mathrm{G}\right)$ do not exist. We have extended the stability analysis to numerical models of solar magnetic flux tubes that include all tension forces and allow for a non-vanishing internal atmosphere.

\section{The Stability Criterion}

Consider a magnetic flux tube as depicted in Figure 1. In magnetohydrostatic equilibrium we have at every point of the surface $S$

$$
p_{i}+\frac{B^{2}}{8 \pi}=p_{e}
$$




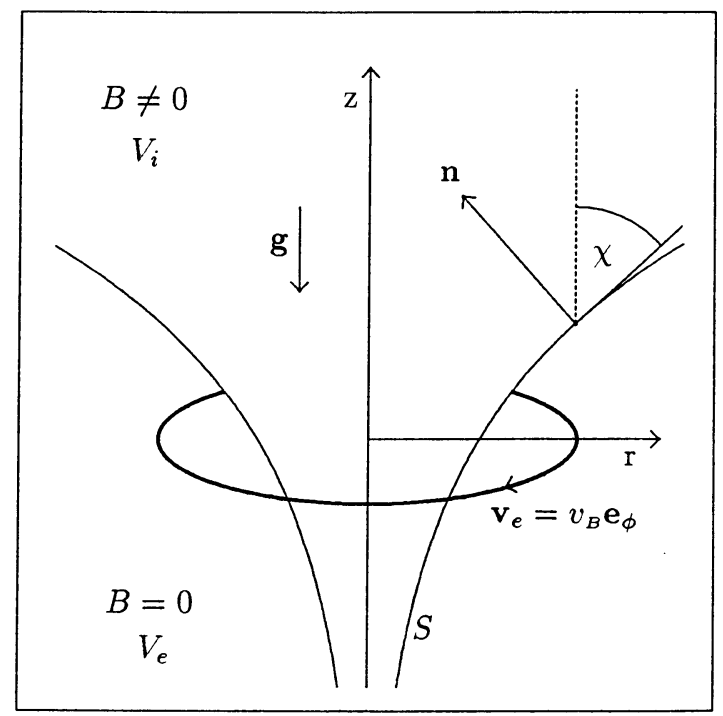

Fig. 1. Geometry of the models considered in this study. $S$ is the surface of the tube, $V_{i}$ is the ("internal") magnetic gas volume, $V_{e}$ the ("external") non-magnetic volume in rotation about the tube axis ( $z$-axis) at velocity $v_{B}$. Gravitational acceleration, $g$, the normal, $\mathbf{n}$, and angle, $\chi$, of the boundary to the vertical are indicated.

where $p_{e}$ and $p_{i}$ are external and internal gas pressures and $B$ is the magnetic field strength at the tube surface. The system is stable to small surface displacements if at any point along $S$ (Bernstein et al. 1958; Frieman and Rotenberg 1960)

$$
\mathbf{n} \cdot\left[\nabla\left(p_{i}+\frac{B^{2}}{8 \pi}\right)-\nabla p_{e}\right]>0
$$

where $\mathbf{n}$ is the unit normal pointing into the tube (see Fig. 1). We apply this criterion to a cylindrically symmetric, untwisted flux tube embedded in a vertically stratified atmosphere and surrounded by a purely azimuthal flow $\mathbf{v}=v_{B} \mathbf{e}_{\phi}$ (the "whirl flow"). Using the respective momentum equations we substitute the gradients in the stability criterion (1) and obtain as a condition for stability (Bünte et al. 1992)

$$
-\left.\frac{1}{4 \pi} B_{z} \frac{\mathrm{d} B_{r}}{\mathrm{~d} z}\right|_{S}+\rho_{e} v_{B}^{2} \frac{1}{R}>0,
$$

where $B_{r}$ and $B_{z}$ are the magnetic field components at the tube boundary, $\rho_{e}$ is the external density and $R$ is the radius of the tube. The index $S$ indicates that the derivative is evaluated along the surface $S$. From this criterion it follows that: (1) the system is susceptible to interchanges if the radial component of the magnetic field increases with height along the boundary, and (2) the surface can be stabilized by an external whirl flow surrounding the tube. In a given atmosphere (i.e., prescribed interior and exterior atmospheres) the only free parameter determining the structure of a flux tube is the total magnetic flux $\Phi$. In the following we compute a magnetic flux tube and determine the $v_{B}$ value which marginally stabilizes 

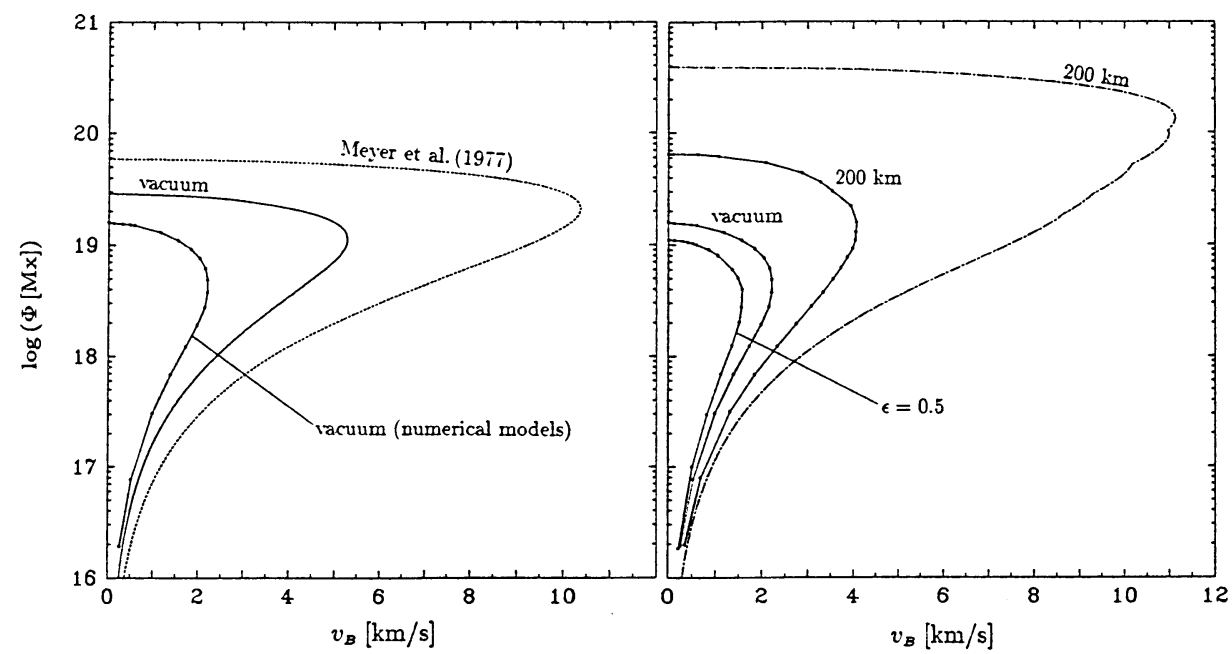

Fig. 2. Comparison of numerical models with thin tubes. Left: stability curves for evacuated tubes. - $\bullet-$ : numerical model tubes, embedded in a Spruit/VAL atmosphere; --: corresponding thin tube result; - - -: thin tube result for the standard atmospheric model of Meyer et al. (1977) (see section 3.1). Right: stability curves for flux tubes with internal atmospheres. --: partially evacuated, "isothermal" tubes with $\epsilon=p_{i} / p_{e}=0.5$, completely evacuated tubes, and tubes whose internal atmosphere corresponds to the external atmosphere shifted downwards by $200 \mathrm{~km} ;-\cdot-$ : result for thin tubes in the latter case (see section 3.2).

the tube. This corresponds to one point $\left(v_{B}, \Phi\right)$ in a flux vs. whirl velocity diagram. By repeated application to tubes of various fluxes we thus obtain a "stability curve" which separates stable and unstable regimes. The whirl velocity can serve as a "measure" of the instability in the sense that flows of moderate velocities, up to $2-3 \mathrm{~km} \mathrm{~s}^{-1}$ in the case of the Sun, appear likely to occur (Nordlund 1985), while much higher values indicate "severe" cases that are unlikely to be stabilized by this mechanism. A twist of the field would help to stabilize the tube. However, the twists needed for the stabilization of the interchange instability are in general so large that a quasistatic equilibrium is no longer possible (Bünte et al. 1992). Therefore, in the following we will concentrate on untwisted tubes.

\section{Results of the Stability Analysis}

We have calculated magnetic flux tubes embedded in a Spruit-VAL atmosphere (Spruit 1977) using the code of Pizzo (1990). First we have concentrated on evacuated tubes to isolate the influence of the magnetic tension forces in comparison with the thin tube results. In a second step we have considered two different cases: (1) the internal pressure is a constant fraction, $\epsilon$, of the external one at equal geometric height, referred to as the "isothermal" case, and (2) the internal atmosphere is identical to the external one, but shifted downwards by $200 \mathrm{~km}$. 


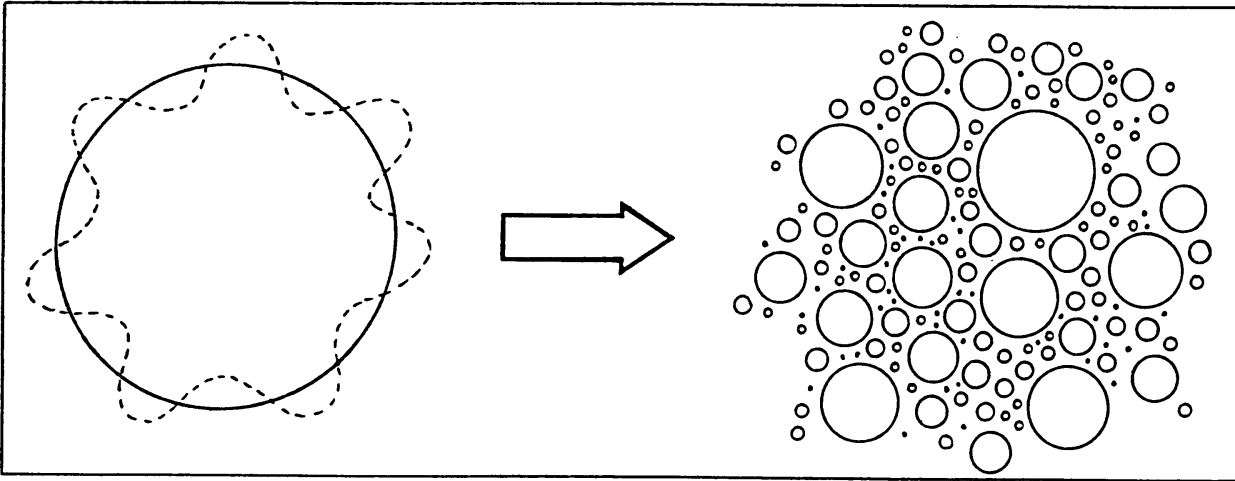

Fig. 3. Sketch of the shredding scenario: The undisturbed (-) cross section of a magnetic flux tube is deformed (- - ) due to fluting (left). If the system is interchange unstable the dashed contour is further distorted, leading to a fragmentation of the original tube (right). The smaller a fragment is the more efficiently it is heated by the radiation from the surrounding atmosphere.

\subsection{The influence of MAgnetic TENSION FORCES}

The results are summarized in the left diagram of Figure 2. The stabilization of thin tubes requires much higher whirl velocities than those of numerical model tubes including all tension forces. If the standard atmospheric model of Meyer et al. (1977) is used, the necessary velocities are even larger. Their atmosphere has a somewhat steeper temperature gradient in the critical layer at $\tau_{5000}=1$. This demonstrates the great sensitivity of the stability criterion to changes in the atmospheric parameters.

\subsection{The influence of an internal atmosphere}

The results are summarized in the right diagram of Figure 2. An internal atmosphere can have either a stabilizing effect (in the isothermal case) or a destabilizing effect (in the shifted case) as compared to the vacuum case. For the latter case the thin tube result is also plotted (dot-dashed curve). Again, thin-tubes require much higher whirl velocities for the stabilization of the interchange instability than "real" tubes.

\section{Discussion}

We have shown that realistic (numerical) models of solar magnetic flux tubes are much easier to stabilize against fluting than thin-tube structures which do not take magnetic tension forces into account. A possible stabilization mechanism is whirl flows. Their velocities lie well within the range of values $\left(2-3 \mathrm{~km} \mathrm{~s}^{-1}\right)$, reported from numerical simulations of solar granulation (Nordlund 1985), and are up to $60 \%$ lower when magnetic tension is included than for the thin-tube case. An internal atmosphere can either have a stabilizing or destabilizing effect depending upon the details of the assumed gas pressure stratification. Nevertheless, in the following 
cases the interchange instability might set in and lead to the shredding of flux tubes - (1) if strong enough whirl flows are absent, or (2) if other flute instabilities are excited, e.g., the MHD Kelvin-Helmholtz instability driven by relative motion.

As shown in Figure 3, fluting-induced shredding of flux tubes produces ever smaller magnetic features. The smaller these features become the more effectively they are heated by radiation from the surrounding photosphere. The measurable field strength should then decrease because of two effects: (1) The increase in temperature shifts the $\tau_{c}=1$ surface to higher atmospheric levels, i.e., to lower field strengths (Steiner and Pizzo 1989), and (2) the heating increases the internal gas pressure and leads to an expansion of the tube. The latter effect could be denoted as the "inverse process" of the convective collapse. In fact, radiative effects have been found to reduce the efficiency of the convective collapse in the concentration of magnetic fields (Venkatakrishnan 1986).

Consequently, the onset of the instability leading to a rearrangement of magnetic flux may be tested by measuring the field strength as a function of time. Since very exact measurements of the field strength are required, they are best carried out using the $g=3$ line at $1.5648 \mu \mathrm{m}$. Even better constraints can be set by combining time series of Stokes $V$ observations of this line with Stokes $V$ observations of a temperature diagnostic in the visible (e.g., the well-studied set of lines around $5,250 \AA$ ). The expected signature is a drop in the field strength by a few hundred $G$ over a time-scale of a few minutes coupled to a warming of the magnetic atmosphere. Also, if a compact flux tube with a diameter of a few hundred $\mathrm{km}$ is broken up into a number of fragments having a range of sizes and, consequently field strengths, then the $\sigma$-components of the $V$ profile of the $1.5648 \mu \mathrm{m}$ line should become anomalously broadened.

\section{Acknowledgements}

The work of M.B. was supported by grant No. 20-31'289.91 of the Swiss National Science Foundation, which is gratefully acknowledged.

\section{References}

Bernstein, I. B., Frieman, E.A., Kruskal, M.D., Kulsrud, R. M.: 1958, Proc. R. Soc. London A 244, 17.

Bünte, M., Steiner, O., Pizzo, V.J.: 1992, Astron. Astrophys. (submitted).

Frieman, E. A., Rotenberg, M.: 1960, Rev. Mod. Phys. 32, 898.

Meyer, F., Schmidt, H.U., Weiss, N.O.: 1977, Mon. Not. R. Astron. Soc. 179, 741.

Nordlund, A.: 1985, Solar Phys. 100, 209.

Pizzo, V.J.: 1990, Astrophys. J. 365, 764.

Schüssler, M.: 1984, Astron. A strophys. 140, 453.

Spruit, H.C.: 1977, Ph.D. Thesis, Univ. Utrecht.

Steiner, O., Pizzo, V.J.: 1989, Astron. Astrophys. $211,447$.

Venkatakrishnan, P.: 1986, Nature 322, 156.

Vernazza, J.E., Avrett, E.H., Loeser, R. (VAL): 1976, Astrophys. J. Suppl. 30, 1. 